\title{
Personal Cloud Storage using Raspberry Pi
}

\author{
Fairuz Rauf \\ Faculty of Communication, \\ Visual Art and Computing \\ Universiti Selangor \\ Malaysia
}

\author{
Maalik Ithing \\ Faculty of Communication, \\ Visual Art and Computing \\ Universiti Selangor \\ Malaysia
}

\author{
Zuraidy Adnan \\ Faculty of Communication, \\ Visual Art and Computing \\ Universiti Selangor \\ Malaysia
}

\begin{abstract}
The project is developed for user need the personal cloud storage service using Raspberry pi. The purpose of developing the system is to explore the Raspberry pi 3 act as server and open source software for develop it as personal cloud storage services. This project has been done by research the developing it like same information that gain through research and few functions like LINE notify add on it. The system will be offering cloud storage like commercial cloud service that already existing. Personal cloud storage offers for free services, synchronizing devices and sharing content. Personal cloud storage using Raspberry pi allows to synchronize local folder with servers in the cloud with accessing internet. All the process must be done by login into the system. This project also let user to mount USB or external storage and can access through it.
\end{abstract}

\section{General Terms}

Security, Cloud services, Storage

\section{Keywords}

Raspberry Pi, LINE, Notification, Storage, OwnCloud

\section{INTRODUCTION}

Personal cloud storage using Raspberry pi services will allow to synchronize local folders with raspberry that act as servers in the cloud. Personal cloud storage offering for free services, synchronizing devices and sharing content. Personal cloud storage also can be referring the way to access software and storing data in the cloud representation of the internet. It is also an excellent way to make sure all your files are accessible at anywhere you go. Raspberry pi is also best alternative to make personal cloud storage because it confirms about the security. The reason is because, without installing any additional software, Raspberry pi able to use Secure Shell (SSH). Secure Shell (SSH) is known as UNIX-based command interface and protocol for securely getting access to a remote computer. As well as, SSH allows you to connect to your server securely and perform Linux command-line operations. Due to that, it proves that can easily connect our Raspberry pi that act server from other computer such as Linux computer, Mac, and others Raspberry pi. Furthermore, with enable SSH is the alternative way can make sure all data it keeps safe between two computers when access of internet. However, in this project need to make raspberry that acts as server personal cloud storage. When someone access into SSH command line, they may can change the folder, access all file in server or shutting down the server without our permission. Even SSH in enable and provide username and password but how to make sure it safety enough because that attacker may try double effort to access, with that reason LINE Notify is adding on this project. It works when developer or others access to SSH in command line then send notify on LINE application. By this Notify, developer can notice when attacker try access in server.

Personal cloud storage is a cheap alternative cloud storage while it also the main requirement for this project. Raspberry $\mathrm{Pi}$ is the suitable hardware to develop a small, power independent, portable and lightweight to develop personal cloud storage. Raspberry Pi is like microcomputer created by the head of Raspberry Pi foundation that can support with open source software. The Raspberry Pi 3 model will be used in this project is the new generation and it because faster and more powerful than its predecessors. In this project, need to using open source like Owncloud. OwnCloud provides a safe, secure, and compliant file synchronization and sharing solution on servers that user control. Plus, user can share one or more files and folders on their raspberry pi, and synchronize them with ownCloud. The service will available to users over an internet. It allows the user to store files online and user can access them from anywhere via internet.

\section{OVERVIEW}

\subsection{Problem Statement}

i. Security issues regarding the existing cloud storage that synchronous by social media account that will exploit by intruder Most of user using same email and password for online media that will let intruder try to access your cloud storage and thief data.

ii. Cloud services provider gives limited cloud storage. An existing cloud storage only provide a limited storage and need to pay if want earn an extra storage

iii. There is no notification to alert user if someone is trying to access and modify on raspberry pi that act as the server for personal cloud storage The previous case study did not mention about notification on Raspberry pi and how to configure it.

\subsection{Objectives}

i. To develop a personal cloud storage using Raspberry pi that only using password and username with added security features involving encryption that provide by OwnCloud.

ii. To add-on some features in cloud storage where user can expand storage space using their own secondary storage devices without any charge.

iii. To configure an alert notification through LINE mobile application to mobile phone user. 


\subsection{Scope and Limitations}

The system develops cloud using Raspberry Pi 3 which supported by open source and wifi. This system can only be accessed by developer that act as user in this system because has set their own password and username to login into the system and only can be access at everywhere with connecting in a same local network (LAN). An open source that act as cloud storage platform named as Owncloud. An Owncloud able to change background for interface login and a few functions in it but developer change interface background, slogan and logo.

\section{LITERATURE REVIEW}

The literature will be reviewed based on cloud storage features and functions that capability for nowadays. From the literature review, it is found that many company already provided the cloud services but have some differences features among of it. While, also found that Raspberry pi can applied in the way to make a personal cloud storage. Raspberry pi can be configuring with proper guidelines and make own cloud storage.

Public cloud allows users access to the cloud via interfaces using web browsers but users need to pay only for the time duration they use the service. This can be compared to the electricity system which user receive at our homes. User pay only for the amount of that we user. The same concept applies here. This helps in reducing the operation costs on IT expenditure. However public clouds are less secure compared to other cloud models as all the applications and data on the public cloud are more prone to malicious attacks. The solution to this can be that security checks be implemented through validation on both sides, by the cloud vendor as well as the client. Also both the parties need to identify their responsibilities within their boundaries of operation. (Wang, Ren, Lou, \& Li, 2010)

A private clouds operation is within an organization's internal enterprise data centre. The benefit is easier to manage security, maintenance, upgrades and also provides more control over the deployment and use. Private cloud also can be compared to intranet. Compared to public cloud where all the resources and applications were managed by the service provider, in private cloud these services are pooled together and made available for the users at the organizational level. The resources and applications are managed by the organization itself. Security is enhanced here as only the organizations when user need access to the private cloud (Wang, Ren, Lou, \& Li, 2010).

Hybrid cloud is a combination of public cloud and private cloud. In this model a private cloud is linked to one or more external cloud services. It is more secure way to control data and applications and allows the party to access information over the internet and it also enables the organization to serve its needs in the private cloud. Then, if some occasional need occurs it asks the public cloud for intensive computing resources (Wang, Ren, Lou, \& Li, 2010).

Advantages of using cloud storage includes easy management, cost reduction and accessibility. Method to access cloud storage services includes software as a Service (SaaS) (Malik \& Nazir, 2012), Platform as a Service (PaaS) and Infrastructure as a Service (IaaS).

Following table are three major provider of cloud storage:
Table 1: Comparison on existing cloud storage

\begin{tabular}{|l|l|l|l|}
\hline \multicolumn{1}{|c|}{ Type } & \multicolumn{1}{|c|}{ OneDrive } & \multicolumn{1}{c|}{ Dropbox } & \multicolumn{1}{c|}{ Google Drive } \\
\hline $\begin{array}{l}\text { File size } \\
\text { restrictions }\end{array}$ & $10 \mathrm{~GB}$ & $\begin{array}{l}\text { 10GB with website, } \\
\text { none with Dropbox apps }\end{array}$ & $5 \mathrm{~TB}$ \\
\hline Free storage & $5 \mathrm{~GB}$ & $2 \mathrm{~GB}$ & $15 \mathrm{~GB}$ \\
\hline $\begin{array}{l}\text { Earn extra } \\
\text { free storage? }\end{array}$ & No & Yes & No \\
\hline Paid plans & $\$ 2 /$ month for 50GB & $\$ 10 /$ month for 1TB & $\begin{array}{l}\text { \$2/month 100GB, } \\
\text { \$10/month for } \\
1 \mathrm{~TB}\end{array}$ \\
\hline $\begin{array}{l}\text { OSes } \\
\text { supported }\end{array}$ & $\begin{array}{l}\text { Windows, Mac, } \\
\text { Android, iOS, } \\
\text { Windows Phone }\end{array}$ & $\begin{array}{l}\text { Windows,Mac,Linux, } \\
\text { Android,iOS,Windows } \\
\text { Phone,BlackBerry, } \\
\text { Kindle Fire }\end{array}$ & $\begin{array}{l}\text { Windows, Mac, } \\
\text { Android, iOS }\end{array}$ \\
\hline
\end{tabular}

OneDrive is one of Microsoft's storage option for those who uses Windows 8 and 10 have OneDrive built into their operating system, where it shows up in the file explorer next to all of the files on user computer's hard drive. However, anyone can use it on the Web by downloading a desktop app for Mac and earlier versions of Windows, or the OneDrive Android, iOS, Windows Phone and Xbox apps. With using OneDrive, user can store any kind of file in the service, including photos, video and documents, and then access them from any of their Windows PCs or mobile devices. The service organizes files by type for user, so it's easy to find what user need (Sarah Mitroff.2016).

The most popular cloud storage for this current is Dropbox because it is reliable, easy to use and a breeze to set up. All files live in the cloud and user can get to them at any time from Dropbox's website, desktop applications for Mac, Windows and Linux or the iOS, Android, BlackBerry and Kindle Fire mobile apps. User can store any kind of file in Dropbox, by either uploading to the website or adding it with the desktop apps. Those apps live in user file system, hence that user can easily move files from your computer to the cloud and vice versa by dragging and dropping them into your Dropbox folder. The service automatically and quickly syncs files across all of your devices, so user can access everything, everywhere. Here is no size limit on files user upload to Dropbox with the desktop or mobile apps, but larger files can take several hours to upload, depending on user connection speed.

Google is the best combines a complete set of office tools with cloud storage in Drive. User get a little bit of everything with this service, including a word processor, spreadsheet application, and presentation builder, plus 15GB of free storage space. For those user already have a Google account mean user can already access Google Drive. User just have to head to drive.google.com and enable the service and will get $15 \mathrm{~GB}$ of storage for anything you upload to Drive, including photos, videos, documents and more. However, user have to share that $15 \mathrm{~GB}$ with your Gmail account, photos user upload to Google+, and any documents user create in Google Drive. In addition, user can access any of their files from the Drive Web site then user can also download the Drive desktop app for Mac or PC to manage your files from your computer. Thus, user can organize all of their files in the desktop app, and they will sync with the cloud so user can get to them anywhere.

Cloud storage using Raspberry pi is developing for provide the user has their own cloud storage. This project, Raspberry pi installed with Rasbian and Owncloud are needed. Raspberry pi configures by using language Raspbian in 
command line. Based on that, Owncloud which is free open source give alternatives like the others cloud service platforms. Owncloud also enable users to set up Raspberry pi act as Cloud server. The user can store data on it, and they can retrieve data from any place with access with internet. In addition, Owncloud allow to configure like background image, slogan, mounting external storage that will give more similarly to existing cloud storage interface or has its own interface. Raspberry $\mathrm{Pi}$ project is the best potential to innovation for Computer Science education. The project and the resulting infrastructure enable Computing Science students get experience on building and programming full distributed systems. To further this goal, the $\mathrm{Pi}$ is available for as little as $\$ 25$. The machines are currently available in model $\mathrm{A}$ and $\mathrm{B}$, featuring small differences in RAM size and number of input and output ports. All these machines, however, share many of the properties of Cloud-based servers, such as limited storage and peripheral capability at a smaller scale. The use of an educational computing platform to construct a Cloud environment offers a promising combination of learning and research. At the same moment, ARM-based architectures are currently attracting significant attention as a cost effective alternative for largescale compute environments. Even the current versions of the Raspberry Pi have not been designed specifically for this purpose, the potential for further decreasing the cost of individual boards makes the architecture particularly attractive (Tso, White, Jouet, Singer, \& Pezaros, 2013).

Raspberry Pi is a small, powerful, and cheap and education oriented computer. It can operate in the same way as a standard PC but requiring a keyboard for command entry, a display unit and a power supply. This credit card-sized computer with many performances and is perfect platform for interfacing with many devices. The vast majority of the system's components, it is central and graphics processing units, audio and communications hardware along with 256 MB for Model A while 512 MB for Model B memory chip, are built onto single component. The Raspberry Pi board contains essential such as processor, graphics chip, RAM and other optional devices like interfaces and connectors for peripherals. The processor of Raspberry Pi is a 32 bit, 700 $\mathrm{MHz}$ system on a Chip, which is built on the ARM11 architecture and can be overclocked for more power. Plus, SD Flash memory serves as a hard drive to Raspberry Pi's processor. The unit is powered via the micro USB connector while internet connectivity may be via an Ethernet or LAN cable or via an USB dongle (Maksimović, Vujović, Davidović, Milošević, \& Perišić, 2014).

The Raspberry Pi is an interesting aspect could be to make device such as to behave like a server and data storage with installing on it a NoSQL DB, like CouchDB, then connect it to the Arduino on internet so in this way one obtains a real Internet of Thing(IoT)) communication. However, user have to consider that such configuration does not provide a real Cloud service like an Infrastructure as a Service model may. Therefore, user have discarded this hypothesis, because the main object of the project is infinity processing and storage capacity of data from trillions of things and people that are integrated via virtual services in the cloud, for this reason user will employ a Virtual Machine (VM) running inside a private Cloud. The mentioned ability of the Raspberry Pi to store a limited amount of data could be useful for backup functions in case of a short interruption of the communication between the device and the VM (Wright \& Manieri, 2014).

\section{RESEARCH METHODOLOGY}

This project using only a types of data collection. The techniques that had chosen is questionnaire. Questionnaire that aim only at cloud storage users in order to get data user requirement and knowledge about cloud storage. This technique most important to this project because it is based on user preferences and opinion. This project chooses the Rapid Prototyping model to start with the Prototype, Review, and Refine \& Iterate (develop and test). Furthermore, rapid prototype model is very important because it's easy to use in prototype methodology phase.

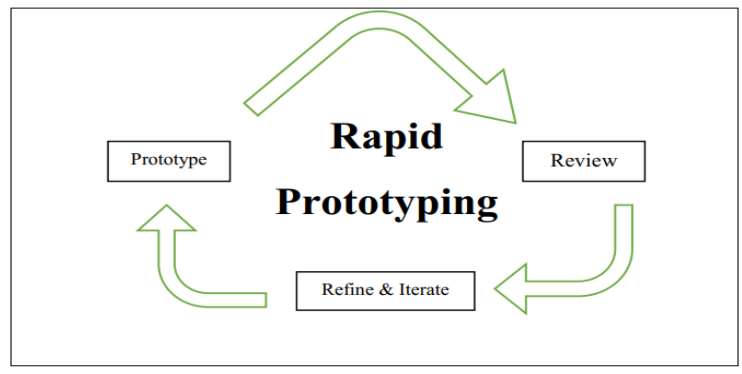

Figure 1: Rapid prototyping

\subsection{Prototype}

In prototyping it is essential to understand the respondent requirements and expectations from the questionnaire. After gathering all the requirements and understanding the user requirement through questionnaire, developer can easily deploy the personal cloud storage of the cloud storage services with selected software and hardware.

\subsection{Review}

In this step, share the prototypes with the random respondent to check whether these are up to their expectations and that everything that they require is incorporated in the prototype.

\subsection{Refine \& Iterate}

After getting the feedback based on random respondent, find the thing need to refinement and clarify. Then, iterate the whole prototyping process until get what the respondent expect. This whole process could take multiple iterations until the prototype is finalized.

\section{DESIGN}

Personal cloud storage architecture is a process of defining the component, modules and data for a cloud storage to fulfil the specific requirement. It implies a systematic and exact approach to design for personal cloud storage. The design to an abstract representation of the data flows, inputs and outputs of the personal cloud storage. The design often conducted via modelling, using a graphical model of the actual personal cloud storage using Raspberry Pi. The main purpose of personal cloud storage design is to create the technical solution to fulfil the functional requirement of the cloud storage. The design also will show the solution to the problem that the developer facing currently and the design will include the process, the flow or order and activity that involve during the personal cloud storage process. 


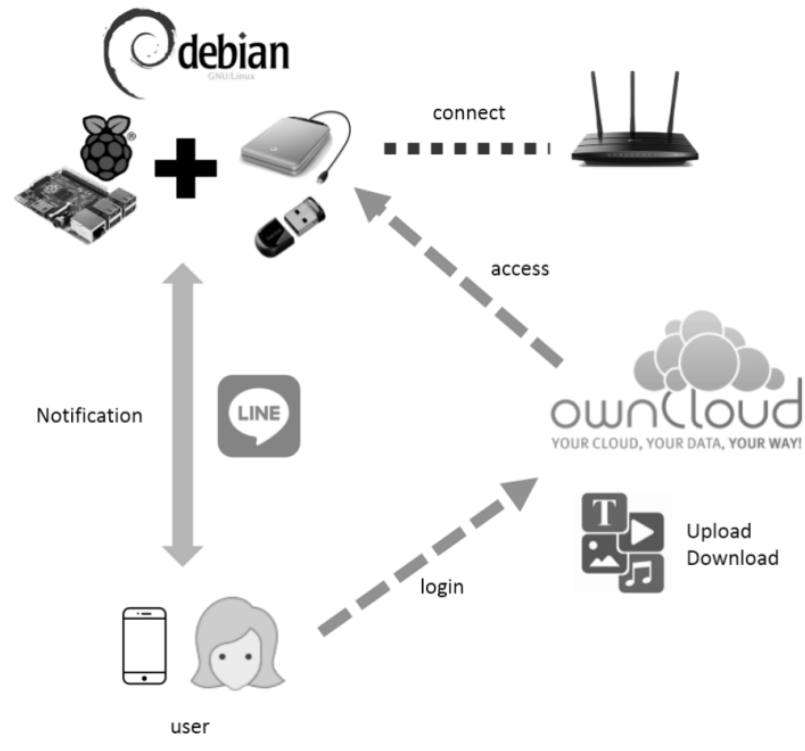

Figure 2: Architecture design

\subsection{Setting up Personal Cloud Storage}

Personal cloud storage using Raspberry pi implementation involves a good of the infrastructure from a server, storage and networking to a security and management software. It is having a five step to keep a personal cloud storage implementation in track.

- Phase 1: Choose the device This phase, it has to choose the devices like Raspberry $\mathrm{Pi}$ and secondary storage devices.

- Phase 2: Setting up Raspberry Pi

- Raspberry pi will configure using basic command line to able Owncloud install on it. This phase need to figure out using coding.

- Phase 3: Monitoring \& Setting up an external drive External drive need to configure setting for mounting and allowing Owncloud to store document, image, audio and video onto it.

- Phase 4: Download and Install Owncloud Developer need to download Owncloud and install. It also provides guider manual to install.

- Phase 5: Setup Owncloud

\section{CONCLUSION}

The personal cloud storage with Raspberry pi provided a lot benefits such as cloud services without any charges, also user can determine their storage space using their own hard disk and added security features involving encryption. With this security features will ensure all data secure. User can have used hard disk with large space hence user can store the large of data on it. Furthermore, user can access to their personal data from any anywhere with this cloud storage services if access internet. This type of cloud services with using Raspberry pi is just a prototype and it implemented with reasonable price, if there are some features need to add on, for instance enhance usability, security or hardware get damaged. For enhance security, developer need to configure and find suitable like create password on hard disk and Raspberry pi. Hence, it become more secure. Then, Raspberry may get damage because of lifetime. To solve this problem, developer provide a cooling system for the porotype to extend the life time hence it would be some cost difficulties for this project. Next, for enhance usability is also including in features for let many users can access into system. As a conclusion, overall project documentation has been proceeding with proper instructions successfully.

The primary purpose of using cloud storage is to store important file, access from anywhere and can back up all files if desktop or Pc has problem that may all files get damaged. For these reasons, personal cloud storage using raspberry pi have been sought to limit or inhibit the disadvantages and weaknesses of current commercial cloud service that used by user. Besides this personal cloud storage supposed to have features like cooling system for the prototype to extend the life time. Plus, provided the good quality housing to protect the prototype such as waterproof.

\section{ACKNOWLEDGMENTS}

Our thanks to all who have involved with this project.

\section{REFERENCES}

[1] Drago, I., Bocchi, E., Mellia, M., Slatman, H., \& Pras, A. 2013. Benchmarking personal cloud storage. Proceedings of the 2013 Conference on Internet Measurement Conference, 205-212.

[2] Drago, I., Mellia, M., Torino, P., Munafo, M. M., Torino, P., \& Sperotto, A. 2012. Inside Dropbox: Understanding Personal Cloud Storage Services. Proceedings of the 2012 ACM Conference on Internet Measurement Conference - IMC'12, 481-494.

[3] Maksimovic, M., Vujovic, V., Davidovic, N., Milosevic, V., \& Perisic, B. 2014. Raspberry Pi as Internet of Things hardware: Performances and Constraints. Design Issues, 3(JUNE), 8.

[4] Tso, F. P., White, D. R., Jouet, S., Singer, J., \& Pezaros, D.P. 2013. The Glasgow raspberry pi cloud: A scale model for cloud computing infrastructures. Proceedings - International Conference on Distributed Computing Systems, (February), 108-112.

[5] Sarah Mitroff: Retrieved date: March 16, (2017), OneDrive, Dropbox, Google Drive and Box: Which cloud storage service is right for you? [Online]. Available at: https://www.cnet.com/uk/how-to/onedrivedropbox-google-drive-and-box-which-cloud-storageservice-is-right-for-you/.

[6] Watanabe Hirofumi. Retrieved date: September 08, (2017), Using LINE Notify to send messages to LINE from the command-line[Online]. Available at: https://engineering.linecorp.com/en/blog/detail/88.

[7] Mitchell McLaughlin. Retrieved date: August 10, (2017), How to set up a personal web server with a raspberry pi[Online]. Available at: https://opensource.com/article/17/3/building-personalweb-server-raspberry-pi-3 